\title{
Note on Japanese Rosaceæ. II.*
}

by

\section{G. Koidzumi.}

1) Rubus pseudo-japonicus, KoIDz. nom. nov.

$R$. triflorus, var. japonicus, Max. in Mél. Biol. VIII. 375 ;O. Kze. Method. p. 133 ;-Matsum. in Bot. Mag. Tokyo, XV. p. 1.

$R$. americanus, var. japonicus, KoIDz. in Bot. Mag. Tokyo. XXIII. p. 175.

$R$. japonicus, Focke. in Bibl. Bot. Heft. 72 (1910) p. 26, (non MAx.).

Nom. Jap. Hime-goyö-ichigo.

Hab. Yezo, Hondō-borealis.

2) Rubus japonicus, Max., O. KzE. 1. c. 130, 155, 156 ;Matsum. 1. c. 1.

R. defensus, Focke. 1. c. 26.

Nom. Jap. Goyō-ichigo.

Hab. Hondō.

3) Malus (Cyclomeles) pumila, Mrrc. Gard. Dict. ed. 8. no. 3, (1768) ;-C. K. Schn. Ill. Handb. Laubholzk. I. (1906). 715.

Pirus pumila, (MILL) K. Koch., Dendrol. I. (1869) 203 ;Asch. et Graeb. Syn. Mitteleurop. Fl. VI. 2. (1906) p. 75.

Pirus malus, L. Sp. P1. ed. 2. p. 686. (p.p.)

Malus malus, BritT. et Brw. Ill. Fl. N-St. Can. II. p 236. var. domestica, (BorkH) C. K. Schn. 1. c. 715.

Malus domestica, Borkn. Handb. Forstb. II. (1803) 1272.

Pirus malus, B. II. domestica, Asch. et GraeBN. 1. c. 77.

* Not. Jap. Rosac. I. in But. Mag. Tokyo. XXIII. p. p. 165-184. 
Nom. Jap. Seiyōolingo.

Hab. Culta.

var. paradisiaca, (MED.) C. K. Scrin. 1. c. 715.

Malus paradisiaca, MEd. Gesch. Bot. (1793), 78.

Malus dasyphylla, Borkн. 1. c. 1271.

Pirus malus, var. mitis, WALL., WG. Linn. XXXVIII. 30 ;Tноме, F1. D. O. S. III. 115.

Pirus malus, var. tomentosa, W. KocH. Syn. F1. Germ. 235, (1837) ;-Ledeb. Fl. Ross. II 96 ;-Max. Mél. Biol. IX. 165 ;Halacsy, Consp. Fl. Graec. I. 539 ;-Ito. Pl. Yoshi. I. 22 ;Fr. et Sav. En. Pl. Jap. II. 349.

Malus communis, a. typica, BECK. Fl. Nied. Öster. (1890), p. 716.

Pirus pumila, e. dasyphylla, Asch. et Graeb. 1. c. 77.

Malus Matsumuræ, var. dulcissima, Kordz. 1. c. 173.

Pirus præcox, Fr. et Sav. 1. c. I. 138 ;-MiQ. Prol. Fl. Jap. 228 (non PALL.).

Nom. Jap. Walingo.

Hab. Culta.

Arbusculus; foliis ellipticis vel elliptico-oblongis, basi saepe cuneato-angustatis, apice saepe subito breve acuta, crebrime serrulatis; petiolis ad $2.5 \mathrm{~cm}$. longis; fructibus minoribus ad $3 \mathrm{~cm}$. latis, maturis luteis et sanguineopictis.

4) Malus (Gymnomeles) floribunda, Sies. Cat. Rasis. 5 (1856) ;-Koenne, Dendrol. 261 ;-Dippex, Handb. Laubholzk. III. $404 ;-C$. K. SchN. 1. c. 721.

Malus Kaido, Sieb. 1. c. (non Wenzig.).

Pirus floribunda, Nichols., W. M. Cycl. Am. Hort. III. 1472.

Pirus Ringo, var. floribunda, WenzIG. Linn. XXXVIII, 38.

Pirus floribunda, Voss. in Vilm. Blumeng. 3. aufl (1896) 277 (non Lindux).

Pirus pulcherrima, Asch. et Graebn. Syn. 1. c. 71.

Pirus spectabilis, MaK. in Bot. Mag. Tokyo. XX. 80, (non Ait.). 
Nom. Jap. Kaidō.

Hab. Culta, (sponte in Kiusiu ?)

Distr. China.

var. Parkmanni m.

Pirus Parkmanni, HoRT.

Malus parkmanni, HoRT.

Nom. Jap. Yaye-kaidō.

Hab. Culta.

5) Malus (Gymnomeles) baccata, Borkн., Roem. Syn. Monogr. III. p. 214 ;-C. K. SchN. 1. c. 720.

var. cerasifera, (SPACH.).

Malus cerasifera, Spach. Hist.Veg. II. (1834), 152.

Pirus cerasifera, Tausch. Flora, XXI. (1834) 714.

Pirus baccata, var. cerasifera, Regel, Gartfl. (1862) p. 202. t. 364. fig. 1, 6.

Malus pumila, var. $\times$ baccata, C. K. Schn. 1. c. 717 .

Pirus baccata $\times$ pumila, Asch. et GraebN. 1. c. 82 .

Nom. Jap. Mi-kaidō.

Hab. Culta.

6) Malus baccata, BorkH. 1. c.

Subsp. Toringo, (SiEB.).

Malus Toringo, Sieb. Cat. Rais. I. (1856), 4 ;-C. K. Schn. 1. c. 723 .

Pirus Toringo, Sieb. in Mrq. Ann. Mus. Lugd. Bat. III. 41. (1856);-K. Косн, Dendrol. I. (1869). 212 ; Asch. et. Graebn. 1. c. 72 .

Pirus mengo, Sieb.

Sorbus Toringo, DE. VRIEs.

Malus Sieboldi, RegeL, Gartf. (1859), 82.

Malus Rivularis var. Toringo. WenzIG. in Linnæa, XXXVIII. 39.

Malus Sargenti, Rehd, in Sargent, Tr. et Shr. I. (1903) p. 71 ;-C. K. Schn. 1. c. 722.

Nom, Jap. Zumi.

Hab. Japonia. 